\title{
Propóleos de Nariño: Propiedades fisicoquímicas y actividad biológica*
}

\section{Propolis from Nariño: Physicochemical properties and biological activity of Propolis}

\author{
SALAMANCA-GROSSO, GUILLERMO'1; OSORIO-TANGARIFE, MÓNICA-PATRICIA²; \\ CABRERA-MONCAYO, JESUS ANTONIO ${ }^{3}$
}

Historial del artículo

Recibido para evaluación: 17 febrero 2021.

Aprobado para publicación: 12 Noviembre 2021.

* Título del proyecto de origen: “Identificación de marcadores moleculares en propóleos colombianos mediante espectrometría de masas con ionización electrospray y valoración de sus propiedades fisicoquímicas". Financiación: Oficina de Investigaciones y Desarrollo Científico a través de la Coordinación General EOCYT- P 410120516, 2018.

1 Universidad del Tolima, Facultad de Ciencias, Departamento de Química, Grupo de Investigaciones Mellitopalinológicas y Propiedades Fisicoquímicas de Alimentos (GIMELLIFISTO). Ph.D. Ciencias Químicas. Ibagué, Colombia. http://orcid.org/00000001-8947-7574.

2 Universidad del Tolima, Grupo de investigaciones Mellitopalinológicas y Propiedades Fisicoquímicas de Alimentos (GIMELLIFISTO). Msc. Microbiología Tropical. Ibagué, Colombia. http://orcid.org/0000-0003-4176-7550.

3 Universidad de Nariño, Facultad de Ciencias, Departamento de Química. Ph.D Ciencias Biomédicas. Pasto, Colombia. https:// orcid.org/0000-0001-5968-226X

Correspondencia: gsalaman@ut.edu.co

Cómo citar este artículo: SALAMANCA-GROSSO, GUILLERMO ; OSORIO-TANGARIFE, MÓNICA-PATRICIA; CABRERAMONCAYO JESUS ANTONIO. Propóleos de Nariño: Propiedades fisicoquímicas y actividad biológica. Biotecnología en el sector agropecuario y agroindustrial, v. 20, n. 1, 2022, p. 152-164. Doi: https://doi.org/10.18684/rbsaa.v20.n1.2022.1863 


\section{RESUMEN}

El propóleo, es un producto natural de consistencia resinosa y cauchosa elaborado por las abejas a partir de exudaciones de plantas. En este trabajo se caracterizaron las propiedades fisicoquímicas, atributos sensoriales y actividad biológica de extractos etanólicos de propóleo de tres diferentes zonas biogeográficas del departamento de Nariño. Muestras de propóleo crudo beneficiados de cada región fueron comparadas, y sus extractos alcohólicos fueron evaluados a través de métodos de separación y técnicas de espectroscopía. La actividad biológica fue evaluada usando cepas bacterianas de Staphylococcus aureus y Escherichia coli. Los análisis cromatográficos revelaron la presencia de metabolitos secundarios con actividad biológica frente a las bacterias estudiadas. Los espectros de infrarrojo y los cromatogramas, exhibieron diferencias entre las muestras, identificados como tipo -O y-B. La fracción fenólica, presentó diferencias significativas para flavonas y flavonoles. Los índices de oxidación fueron inferiores a 5 segundos. En relación a los extractos etanólicos evaluados, éstos mostraron una mayor sensibilidad sobre S. aureus en la técnica de Kirby-Bauer. La investigación realizada aporta al estudio y caracterización de propóleos de esta zona colombiana, ampliando el conocimiento sobre productos de origen natural.

\section{ABSTRACT}

Propolis is natural product processed by honey bees from plant exudations with a resinous and rubber-like consistency. This work examines the physicochemical properties, sensory attributes, and biological activity of propolis ethanol extracts from three different biogeographic zones in department of Nariño, Colombia. Samples of raw propolis obtained from each area were compared, and their alcoholic extracts studied through separation methods and spectroscopic techniques. Biological activity was evaluated using Staphylococcus aureus and Escherichia coli strains. Chromatographic analysis revealed the presence of secondary metabolites with potential biological activity. Infrared spectrum and chromatograms displayed differences between group samples, identified as type -O and -B. Phenolic fraction exhibited significant variations on flavones and flavanols. Oxidation rates were lower than 5 seconds. Evaluated ethanolic extracts exposed greater sensitivity to $S$. aureus under the Kirby-Bauer technique. Present research contributes towards expanding knowledge about natural-origin products like propolis on such Colombian regions.

\section{INTRODUCCIÓN}

El propóleo es reconocido en todo el mundo, ha sido registrado en el Chemical Abstracts Service (CAS) 9009-62-5, como un producto natural elaborado por distintos géneros de abejas, a partir de exudaciones mucilaginosas, gomas, materiales lipofílicos, látex y material resinoso de plantas, que traen a sus colmena y mezclan con secreciones glandulares de la hipofaringe y glándulas cereras. Son de consistencia pastosa, con

\section{PALABRAS CLAVE:}

Actividad biológica; Fenoles y flavonoides; Propóleos colombianos; Propiedades fisicoquímicas; HPTLC; LC-ESI(-)MS/MS.

\section{KEYWORDS:}

Biological activity; Phenols and flavonoids; Colombian propolis; Physicochemical properties; HPTLC; LC-ESI(-)-MS/MS. 
tonalidades castaño, marrón, pardo, rojizo y verde, según el origen botánico y geográfico (Anjum et al., 2019; El-Guendouz et al., 2019). Estos productos han Ilamado la atención en relación a la salud de las abejas y se ha determinado su papel como protector, antiséptico de la colmena y responsable de la salud e inmunidad frente a los agentes infecciosos (Ciftci-Yilmaz et al., 2017; Anjum et al., 2019). En las regiones tropicales, las abejas encuentran diversas fuentes de elaboración, por tanto, su composición química es diferente y variable debido a la riqueza y diversidad de la flora tropical. Existen en la literatura reportes de un sinnúmero de propiedades biológicas sobre los propóleos que actúan como antitumorales, antiprotozoarios, anticancer (Sforcin, 2016; Omar et al., 2017), antihipertensivos, antimicrobianas, antiinflamatorias, antihepatotóxicos y antivirales, entre otras (Veiga et al., 2017; El-Guendouz et al., 2019; Touzani et al., 2019).

Los componentes químicos de los propóleos varían de acuerdo a las fuentes botánicas y a las áreas biogeográficas donde están instaladas las colmenas. Los métodos instrumentales de análisis, actualmente han permitido identificar en matrices de propóleos alrededor de 350 compuestos fitoquímicos (Guzelmeric et al., 2018; Touzani et al., 2019). Considerando la demanda de este tipo de productos, es indispensable establecer los parámetros para identificar el control de calidad de los propóleos de las regiones. El advenimiento y desarrollo de los métodos cromatográficos de análisis han posibilitado el estudio y cuantificación de principios activos presentes en distintas matrices de propóleos (Ahmed et al., 2017; Drescher et al., 2017).

La actividad antibacteriana es la más estudiada, se conoce el efecto de extractos etanólicos de propóleos frente Salmonella typhimurium, Enterobacter aerogenes, Yersinia enterocolitica, Escherichia coli, Staphylococcus aureus, Staphylococcus epidermidis, Bacillus cereus, Listeria monocytogenes, S. saprophyticus, E. faecalis (Zainullin et al., 2018; Seibert et al., 2019; Przybyłek et al., 2019; Veloz et al., 2019). En Colombia, las investigaciones realizadas sobre actividad biológica in vitro de propóleos han sido reportadas desde la región Andina, Boyacá, Caldas, Cundinamarca y Santander (Bastos et al., 2011; Talero et al., 2012; Herrera et al., 2012; Martínez et al., 2012; Velásquez y Montenegro, 2017). Esta actividad frente a patógenos ha sido atribuida a la presencia terpenos, flavonoides y antraquinonas, principalmente.

La producción de propóleos en Colombia ha sido relacionada desde diversas zonas biogeográficas. Algunos trabajos sobre caracterización y cuantificación en muestras de Arauca, Boyacá y San Andrés (Salamanca y Osorio, 2018) y sobre el perfil mineral de muestras de propóleos del Tolima (Salamanca et al., 2004). Adicionalmente, se han reportado trabajos sobre actividad antioxidante de propóleos colectados en Atlántico y Santander (Rodríguez et al., 2012; Herrera et al., 2012). La concepción de propóleos más actualizada entorno a su control de calidad, consiste en la determinación de sus propiedades fisicoquímicas y la estimación de su actividad biológica. En este contexto, el objetivo de este trabajo se ha centrado en el estudio de las propiedades fisicoquímicas, perfilación organoléptica, estimación de componentes minerales, así como la actividad biológica de muestras de propóleos colectados en tres zonas de biogeográficas de Nariño.

\section{MÉTODO}

\section{Reactivos}

Todos los reactivos usados en este trabajo fueron de grado analítico: etanol, metanol, acetato de etilo, acido fórmico y tolueno (Sigma Aldrich de Steinheim, Germany), aminoetildifenilborinato (Fluka Steinheim, Germany), polientilenglicol, ácido sulfirico, hidróxido de potasio (Merck, Hohenbrunn, Germany), patrones de pinocembrina, galangina, pinobanksina, naringenina, ácido cafeico, crisina, quercetina y apigenina (Sigma-Aldrich) y 2,4 dinitrofenilhidrazina (2,4D) (Merck, Alemania). 


\section{Muestras}

Se colectaron nueve muestras de propóleos de tres zonas biogeográficas diferentes, bosque húmedo montano bajo (bh-MB), muy húmedo premontano (bh-PM) y muy húmedo premontano (bmh-PM), para las localidades de Buesaco

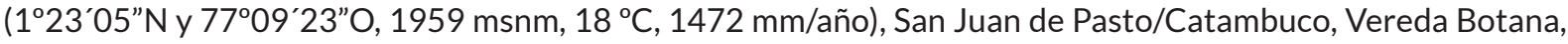

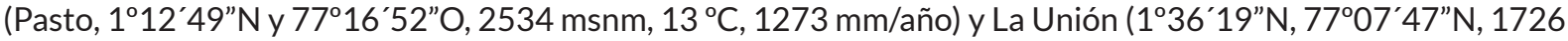
$\mathrm{msnm}, 19^{\circ} \mathrm{C}$ ). Las colectas se realizaron siguiendo criterios de aleatoriedad, con tres niveles de tratamiento y tres repeticiones. La toma de muestras fue realizada usando raspado directamente desde el interior de las colmenas tipo Langstroth removiendo el producto adherido en las caras laterales, tapa, entretapa y alzas. El material se dispuso en frascos ámbar tipo twist off de $250 \mathrm{~mL}$ y almacenado a $-10^{\circ} \mathrm{C}$, hasta el momento del análisis.

\section{Parámetros fisicoquímicos}

Se registró el peso, aspecto, textura, color, olor, sabor e impurezas visibles de los propóleos colectados, así como los puntos de fusión, contenido de humedad, sólidos fijos, cenizas, masas mecánicas, resinas y ceras. Para el análisis de cenizas, se evaluó el contenido mineral mediante espectrofotometría de absorción atómica de calcio, cobre, hierro, magnesio, manganeso, níquel, potasio, sodio y zinc. En el extracto etanólico (EEP) se cuantificó la acidez total, pH, fenoles y flavonoides totales, siguiendo los métodos descritos por Salamanca (2017). La acidez se determinó sobre $50 \mathrm{~mL}$ de agua destilada, libre de $\mathrm{CO}_{2}$ en presencia de fenolftaleína y el volumen total de la solución de hidróxido correspondió a la fracción ácida presente expresada como porcentaje de ácido tartárico. El contenido de fenoles totales, las flavonas, flavonoles, flavanonas y dihidroflavonoles, se determinaron por espectrofotometría UV-Vis. Los fenoles se determinaron siguiendo los protocolos descritos por Baba y Malik (2015) y se expresaron como mg/g de EEP. Las flavonas, flavonoles, flavanonas y dihidroflavonoles, se realizaron usando metodologías descritas por Kasiotis et al. (2017) y Betances-Salcedo et al. (2017), los resultados fueron expresados como mg/g. Las evaluaciones sensoriales se adelantaron siguiendo criterios de Meilgaard (2007), usando escala hedónica sobre seis atributos que fueron evaluados por cinco jueces entrenados. El perfil densitométrico de los extractos se realizó mediante técnicas cromatográficas de alta eficiencia (HPTLC), conforme a Salamanca y Osorio (2018).

Las determinaciones de minerales se realizaron usando espectrofotometría de absorción atómica, haciendo uso de lámparas de cátodo hueco por elemento: Calcio, Hierro, potasio, magnesio y manganeso. El índice de oxidación se midió en extractos alcohólicos (EEP), siguiendo la metodología descrita por Salamanca (2018). La absorbancia específica de los espectros UV-Vis de los EEP, se obtuvo por el método de Miyataka et al. (1997) a la longitud de onda de máxima absorción, usando espectrofotómetro UV-Vis Genesys 10s. Además, los EEP, se evaluaron usando técnicas de espectroscopía FT-IR en la unidad Perkin Elmer Spectrum One spectrometer (Perkin Elmer, USA ${ }^{\mathrm{TM}}$ ), la proyección final de los espectros se realizó haciendo uso del paquete Origin ${ }^{\circledR} 2019$. Los análisis mediante LC-ESI(-)-MS/MS procedieron tras la homogenización mediante ultrasonido, filtración y centrifugado, a través de espectrometro de masas MS/MS, usando un sistema a 7,2T LTQ FT Ultra (Thermo Scientific), dispuesto con chip-based direct infusión, nanoelectrospray ionisation source, la adquisición de datos se realizó en el rango 150-800 m/z, siguiendo la metodología descrita por Sawaya et al. (2011).

\section{Actividad biológica}

La actividad antibacteriana de los EEP, se determinó conforme a los protocolos de Farmacopea Americana USP 24, (Cupull-Santana et al., 2013), operando métodos difusionales sobre bacterias Gram (+) de Streptococcus y bacterias Gram (-) de Escherichiae, las cuales fueron cultivadas en agar tripticasa de soya bajo condiciones aeróbicas por $18 \mathrm{~h}$ a $37 \pm 1^{\circ} \mathrm{C}$. En las determinaciones se usaron los métodos de difusión por pozos y de Kirby-Bauer. El crecimiento de los microorganismos y la difusión del antibiótico se estimaron de acuerdo con el tamaño de las zonas de inhibición a 24, 48 y 72 horas. El mismo procedimiento fue aplicado a un control de etanol al 80 \%, la solución de concentración de $10 \mathrm{mg} / \mathrm{mL}$ fue preparada a partir de la solución estándar de sulfato de estreptomicina $(0,1 \% \mathrm{p} / \mathrm{v})$ en agua destilada estéril con buffer fosfato $\mathrm{pH} 8,0$. 


\section{Estadística}

Las determinaciones analíticas se realizaron por triplicado en cada una de las muestras colectadas. En todos los casos se estimó el promedio, desviación estándar y coeficientes de variación de cada parámetro. Las evaluaciones para el análisis de varianza de una vía se efectuaron haciendo uso del paquete estadístico JMP14 ${ }^{\mathrm{TM}}$. Las evaluaciones densitométricas de los perfiles de cromatografía de capa fina de alta eficiencia (HPTLC) haciendo uso del software libre ImageJ (Wayne Rasband, NIH, USA).

\section{RESULTADOS}

Los propóleos estudiados corresponden a los tipos III, IV y V, que corresponden a las zonas biogeográficas enmarcadas y consistentes con los observados en otras muestras de zonas tropicales (Salamanca, 2017). Los extractos etanólicos de los propóleos de las zonas de estudio presentarón un carácter ácido, las diferencias en este parámetro pueden ser explicadas teniendo como referencia factores climáticos, la oferta floral y el tiempo en el cual las abejas han propolizado las colmenas.

Los promedios para el contenido de cenizas entre muestras presentan diferencias significativas, siendo mayor en los propóleos de Pasto con 1,70 \%. Los tenores de cera oscilaron entre 10,1 y 19,0\%. La mayor proporción de masas mecánicas se observaron en las muestras de Pasto, respecto de las otras dos zonas. Las resinas, son indicativas de la calidad del producto en términos de la actividad antioxidante, sus valores oscilaron entre 50,2 y $60,3 \%$ (Cuadro 1).

Cuadro 1. Parámetros fisicoquímicos asociados a muestras de propóleos de tres zonas biogeográficas de Nariño.

\begin{tabular}{|c|c|c|c|c|c|}
\hline \multirow{2}{*}{ Parámetros } & \multirow{2}{*}{ Unidades } & bh-PM & bh-MB & bmh-PM & \multirow{2}{*}{ Valores guía } \\
\hline & & Buesaco & S.J. Pasto & La Unión & \\
\hline Punto de fusión & ${ }^{\circ} \mathrm{C}$ & $63,0(1,00)^{a}$ & $59,0(1,00)^{b}$ & $65,0(1,00)^{a}$ & $60-100^{\circ} \mathrm{C}$ \\
\hline Cenizas & \multirow{4}{*}{$\mathrm{g} / 100 \mathrm{~g}$} & $0,81(0,27)^{a}$ & $1,70(0.30)^{c}$ & $0,74(0,10)^{a, b}$ & - \\
\hline Cera & & $10,1(0,70)^{a}$ & $11,7(3,40)^{a, b}$ & $19,0(2,00)^{c}$ & $<25 \%$ \\
\hline Resinas & & $60,3(7,40)^{a}$ & $50,2(2,60)$ & $54,8(7,54)$ & $50-60 \%$ \\
\hline Masas mecánicas & & $27,3(6,60)^{a}$ & $38,2(3,10)^{b}$ & $29,0(6,20)^{a}$ & $<25 \%$ \\
\hline Acidez (A. tartárico) & $\mathrm{mg} / 100 \mathrm{~mL}$ & $6,60(2,36)^{a}$ & $9,10(1,26)^{b}$ & $16,4(0,32)^{c}$ & - \\
\hline $\mathrm{pH}$ & - & $4,20(0,13)^{a}$ & $4,40(0,11)^{b}$ & $4,10(0,07)^{a}$ & - \\
\hline
\end{tabular}

Letras iguales en la misma fila corresponden a valores comparables entre promedios observados en muestras. Desviación estándar (ds).

Las determinaciones de minerales, son menos frecuentes en muestras de propóleos y exponen variabilidad en el perfil de los elementos analizados. Los promedios observados presentaron menor variación para el contenido de hierro en las muestras de Pasto, frente a los de la Unión y Buesaco. Los elementos más representativos en todos los casos correspondieron a sodio y potasio (Cuadro 2 ).

Cuadro 2. Componentes de la fracción mineral de muestras de propóleos de tres zonas biogeográficas de Nariño.

\begin{tabular}{|c|c|c|c|c|c|c|c|}
\hline \multirow{2}{*}{ Mineral } & Buesaco & La Unión & S.J. Pasto & \multirow{2}{*}{ Mineral } & Buesaco & La Unión & S.J. Pasto \\
\hline & & $\mathrm{mg} / \mathrm{Kg}$ & & & \multicolumn{3}{|c|}{$\mathrm{mg} / \mathrm{Kg}$} \\
\hline Calcio & $66,3(21,2)$ & 1298 (909) & $2050(950)$ & Sodio & $436,1(15,4)$ & $340,5(25,8)$ & $431,1(77,7)$ \\
\hline Hierro & $312(59,8)$ & $357(57,9)$ & $101,0(10,0)$ & Zinc & $72,9(8,0)$ & $110(32,5)$ & $35,0(11,0)$ \\
\hline Potasio & $1529(294)$ & $1989(632)$ & $1832(345,5)$ & Sodio/Potasio & 0,285 & 0,171 & 0,235 \\
\hline Magnesio & $47,3(12,9)$ & $359,6(43,3)$ & $763,6(169,2)$ & Cobre & ND & ND & ND \\
\hline Manganeso & $11,6(4,40)$ & $91,2(18,6)$ & $5,64(0,26)$ & Niquel & ND & $1,61(1,27)$ & ND \\
\hline
\end{tabular}

Desviación estándar (ds). ND: No determinado. 
Los contenidos de fenoles presentaron diferencias importantes entre los grupos de muestras, valores están correlacionados con los promedios observados para flavanonas, flavonas y flavonoles, que también concuerdan con los tiempos de oxidación (Cuadro 3). El parámetro $\mathrm{E}_{1 \mathrm{~cm}}{ }^{1 \%} \mathrm{de}$ la absorción UV-Vis, es otro de los parámetros fisicoquímicos usados para evaluar la calidad de los extractos de propóleos y se relaciona con la actividad biológica de metabolitos secundarios como ácidos fenólicos y flavonoides. Los valores de $\mathrm{E}_{1 \mathrm{~cm}}{ }^{1 \%}$ de las muestras, presentaron valores entre 77 y 370 , siendo menores en los extractos de Buesaco, respecto de los extractos de la Unión y Pasto.

Cuadro 3. Compuestos fenólicos e índices de oxidación de EEP de tres zonas biogeográficas de Nariño.

\begin{tabular}{|c|c|c|c|c|}
\hline Componente & Unidades & Buesaco & La Unión & S. J. Pasto \\
\hline Fenoles & \multirow{3}{*}{$\mathrm{mg} / \mathrm{g}$} & $55,0(4,92)$ & $69,1(4,20)$ & $36,6(5,00)$ \\
\hline Flavonas+Flavonoles & & $17,8(2,90)$ & $17,6(1,42)$ & $7,55(0,43)$ \\
\hline Flavanonas & & $37,1(7,30)$ & $51,5(2,83)$ & $29,1(5,41)$ \\
\hline $\mathrm{E}_{1 \mathrm{~cm}}{ }^{1 \%}$ & - & 84-105 & $106-370$ & $77-103$ \\
\hline Índice de oxidación* & $\mathrm{s}$ & $3,90(0,80)$ & $3,40(1,10)$ & $3,60(0,50)$ \\
\hline
\end{tabular}

(*) En EEP. Valor guía (22 s). s: seguindos. Desviación estándar (ds).

La huella espectral FT-IR de los extractos de propóleos estudiados son característicos (Figura 1), se observaron señales con oscilación y vibración ( $\mathrm{cm}^{-1}$ ) de grupos alifáticos (2930 y 2876), carbonilo (1660-1680), hidroxilo (3200-3650) y anillos aromáticos sustituidos (1500, 1580 y 1600). Las bandas de absorción (3300-3600 cm-1), se relacionaron con grupos - $\mathrm{OH}$ de flavonas hidroxiladas, isoflavonoides con una banda significativa a 1680 propia de grupos carbonilo, a $1620 \mathrm{~cm}^{-1}$, otras señales características se presentaron a $1440 \mathrm{~cm}^{-1}, 1283$ y 1231 $\mathrm{cm}^{-1}$ que son frecuencias de vibración $\mathrm{C}=\mathrm{C}-\mathrm{H}$ propias de anillos aromáticos.

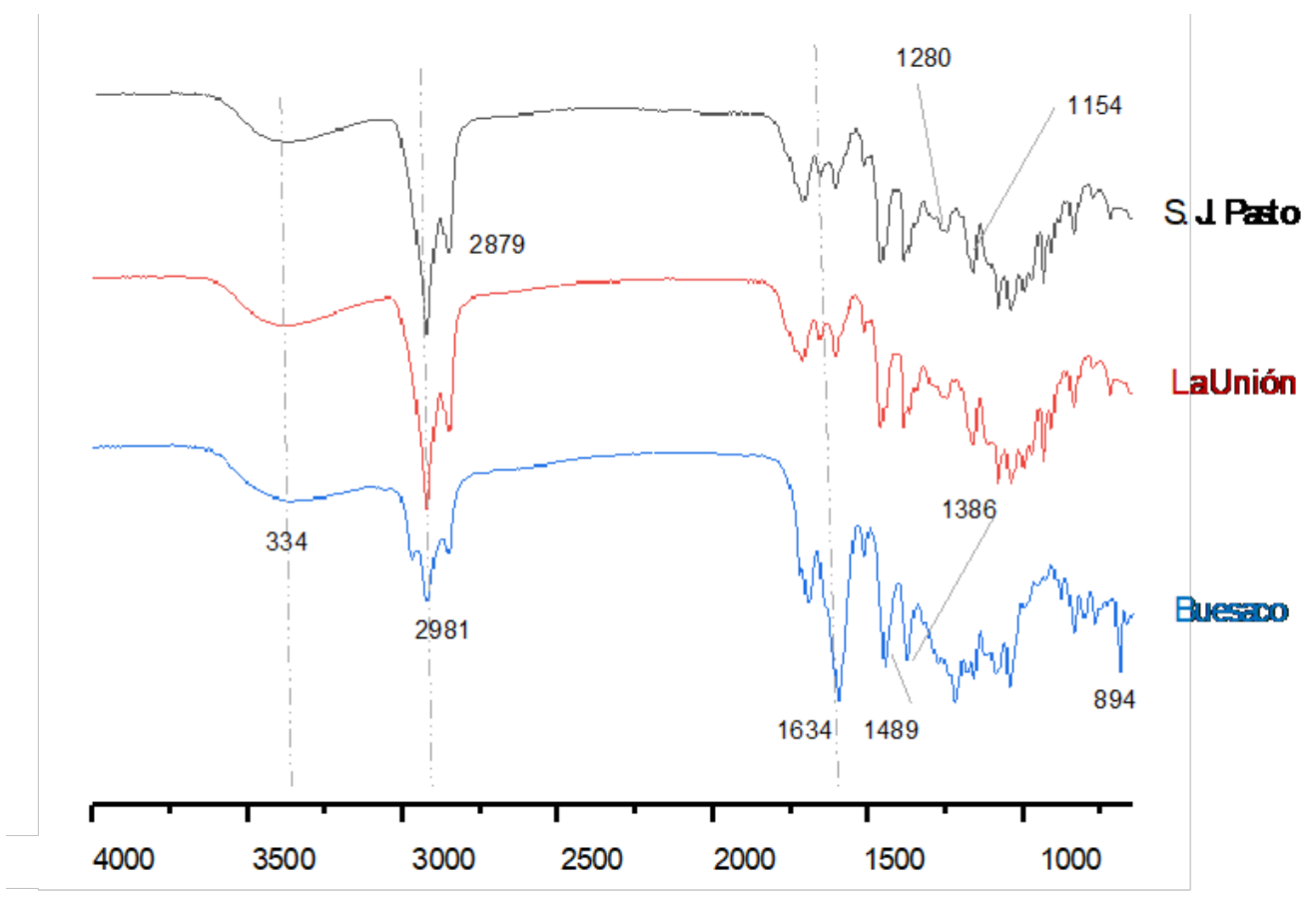

Numerodeonda $\left(\mathrm{cm}^{-1}\right)$

Figura 1. Espectros de infrarrojo (FT-IR) de EEP de tres zonas geográficas de Nariño. 
La inspección visual de los cromatogramas mediante HPTLC, en principio no revelaron diferencias mayores para los $h R_{F}$, sin embargo, se identificaron bandas representativas de compuestos fenólicos, propios de los propóleos de Nariño. Se presentaron 13 metabolitos secundarios que se asocian en los cromatogramas con diferentes tonos de color, así: verde (apigenina, naringenina y crisina), naranja (quercetina) y azul (ácido cafeico, galangina, feruloil y p-derivados del ácido coumarico) y que concuerdan con otros reportes (Guzelmeric et al., 2018). Los análisis densitométricos, presentaron gran similitud en la composición de los EEP de las muestras de Buesaco y La Unión, que difirieron ligeramente respecto de las de Pasto (Figura 2).
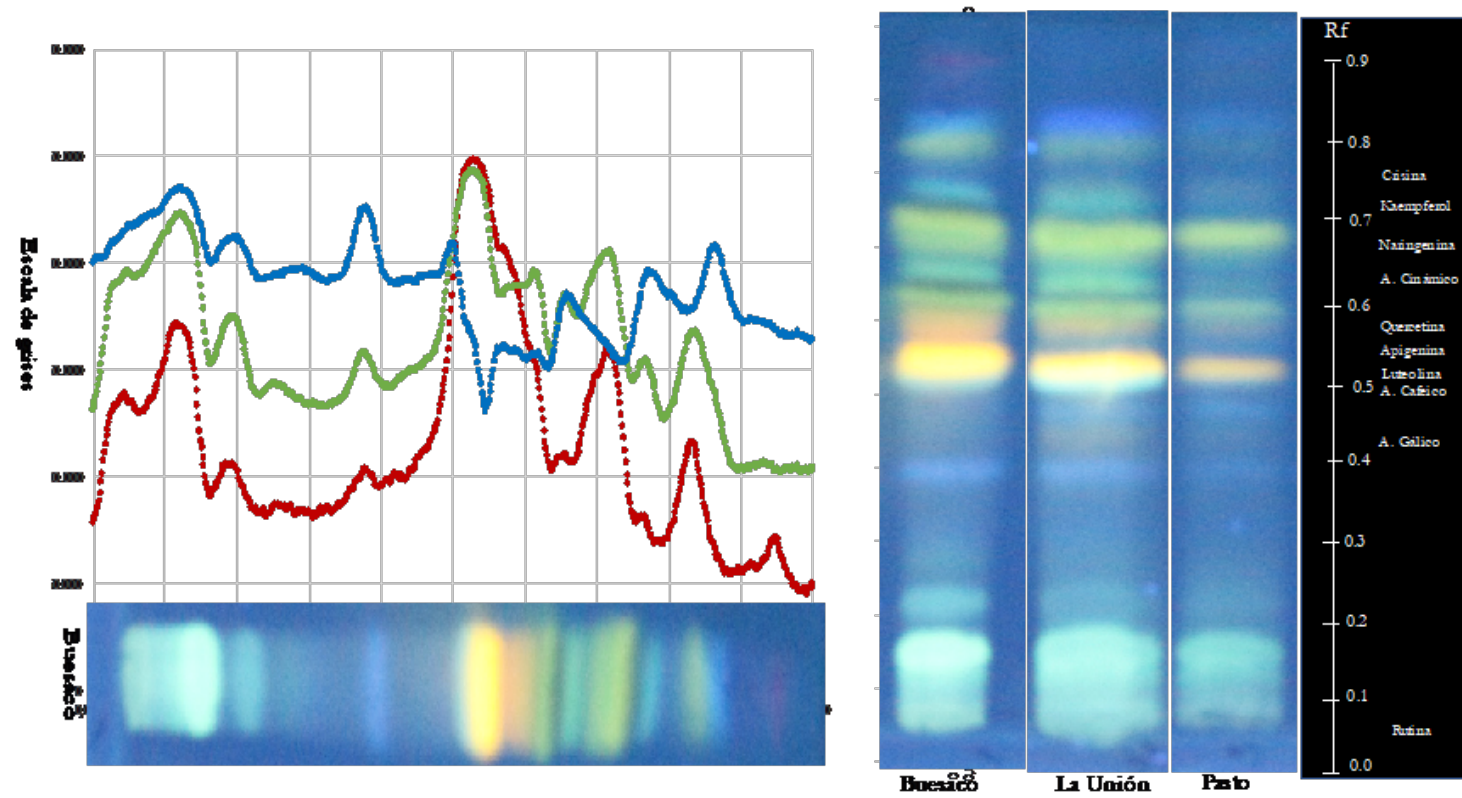

Figura 2. Huella dactilar y perfil densitométrico de cromatogramas de muestras de propóleos de tres zonas geográficas de Nariño.

La huella digital de los cromatogramas mediante técnicas LC-ESI(-)-MS/MS de los extractos de Buesaco y La Unión, presentaron patrones de fragmentación similares (Figura 3). La relación ( $\mathrm{m} / \mathrm{z}$ ), reveló la presencia de acacetina (283), ácido cafeico (179), apigenina (269), fenetil éster de ácido cafeico (179), crisina (253), crisin-5-metilester (267), kaempferol (285), naringenina (271), pinocembrina (255), pinobanksina (271), quercetina (253), luteolin-8-O-glucósido (439) y pinocembrin 8-O-glucósido (439). En los análisis LC-ESI(-)-MS/MS de los EEP, se muestran patrones similares con otros tipos de propóleos reportados en la literatura (Hochheim et al., 2019). Las muestras analizadas presentaron un marcador (m/z: 501), que también ha sido identificado en muestras de Cuba y nordeste de Brasil (Cuesta-Rubio et al., 2007; López et al., 2014; Anjum et al., 2019).

El perfil sensorial de las muestras de Buesaco y La Unión, presentó diferencias en el aspecto, con similitud en los descriptores olfativos y gustativos, que los diferencian de las muestras de Pasto (Figura 4). Las características organolépticas exhibieron aspectos plásticos, maleables, pegajosos y opacos, de tonos marrón con algunas tonalidades verdosas, aromas resinosos y elevada astringencia en muestras de Buesaco y la Unión, que difieren de las de Pasto, que presentaron tonos marrón, de aspecto terroso, resinoso, pegajoso y opaco, sus puntos de fusión mostraron valores similares entre 59 a $65^{\circ} \mathrm{C}$. 
En cuanto a la actividad biológica, se observó un importante efecto inhibidor de los extractos frente a S. aureus y E. Coli. Los halos de inhibición para E. coli y S. aureus observados mediante Kirby-Bauer, revelaron un ligero efecto de sensibilidad de los extractos respecto del método de difusión (Figura 5). El uso de estreptomicina como referencia a $10 \mu \mathrm{g} / \mathrm{mL}$ mostró mayores halos de inhibición en relación a los extractos de las muestras estudiadas. Los extractos de propóleos exhibieron mayor actividad frente a microorganismos Gram (+) que Gram (-), resultados que son similares a los reportados para S. aureus, S. saprophyticus, S.epidermidis y S. thermophilus, (AL-Ani et al., 2018), en tanto que los hallazgos y observaciones con los EEP de Nariño son comparables con los datos reportados por Selvaraj et al. (2018).

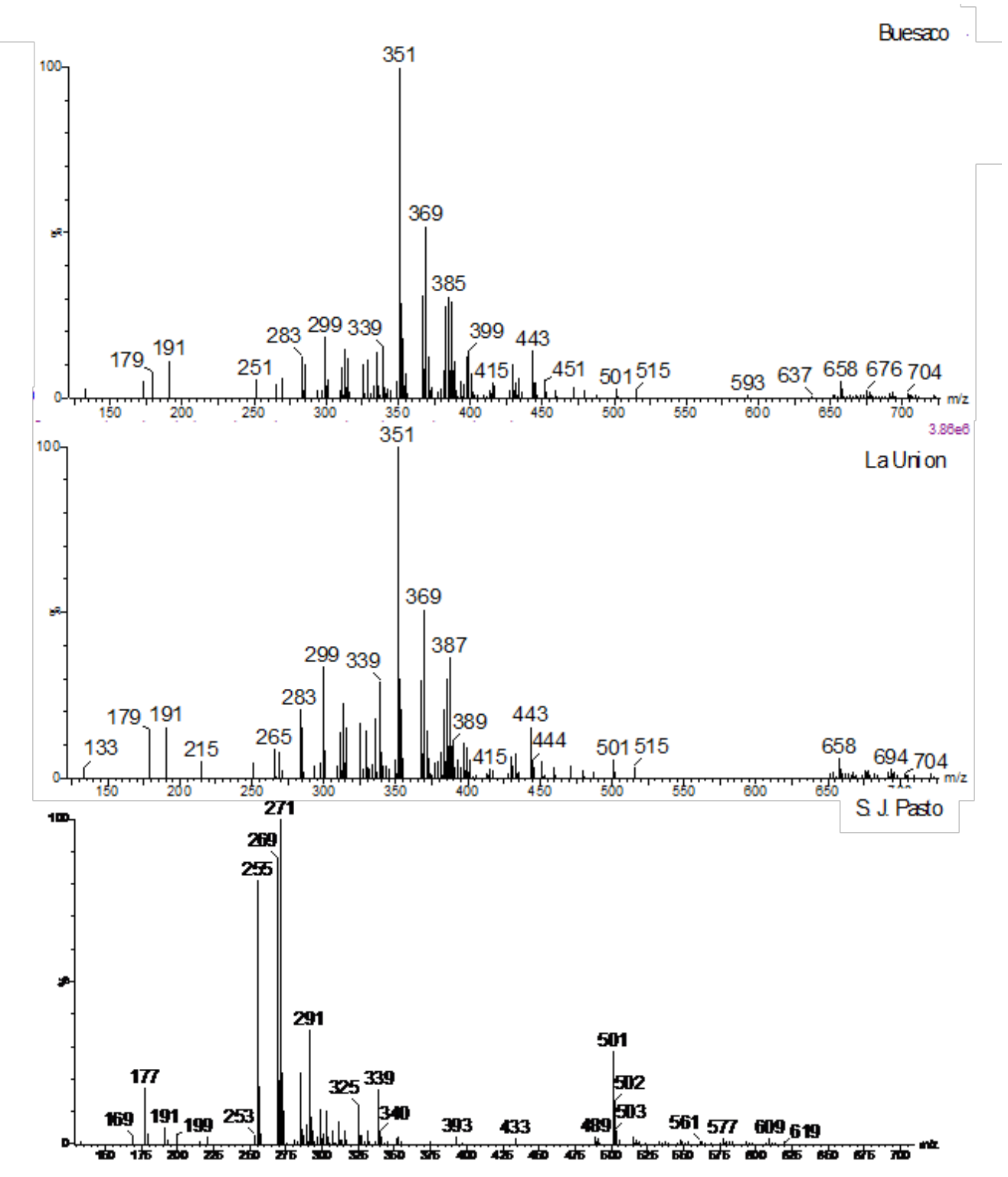

Figura 3. Patrón de fragmentación LC-ESI(-)-MS de muestras de propóleos de Buesaco, La Unión y S.J de Pasto, Nariño. 

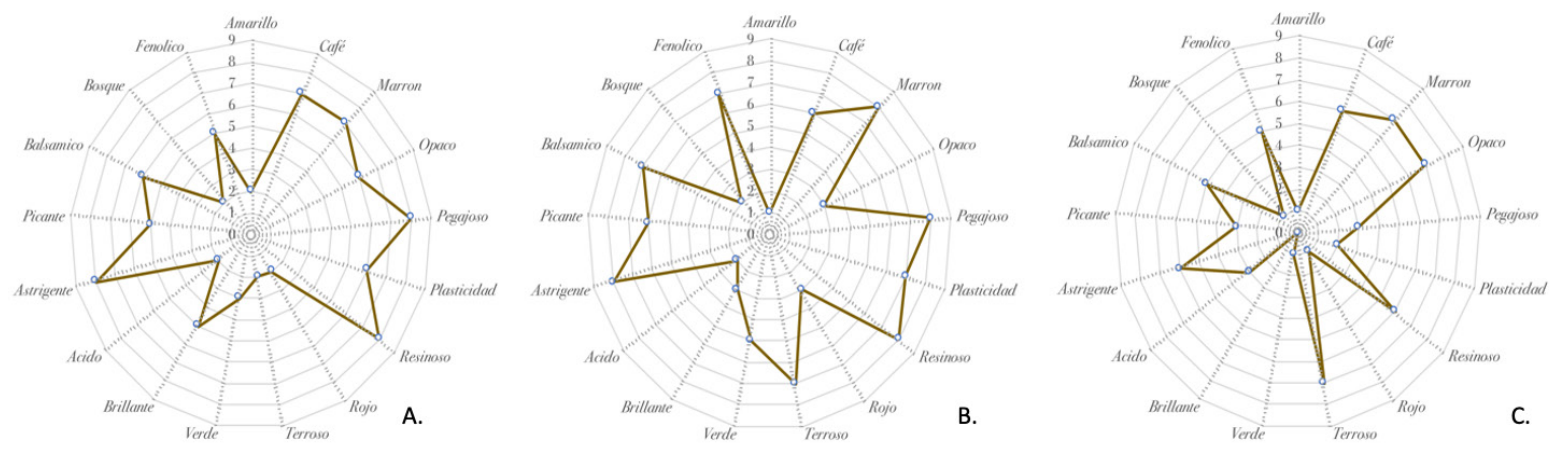

Figura 4. Perfil sensorial de los atributos asociados a muestras crudas de propóleos de las zonas de A. Buesaco, B. La Unión y C. S.J. Pasto, Nariño, Colombia.

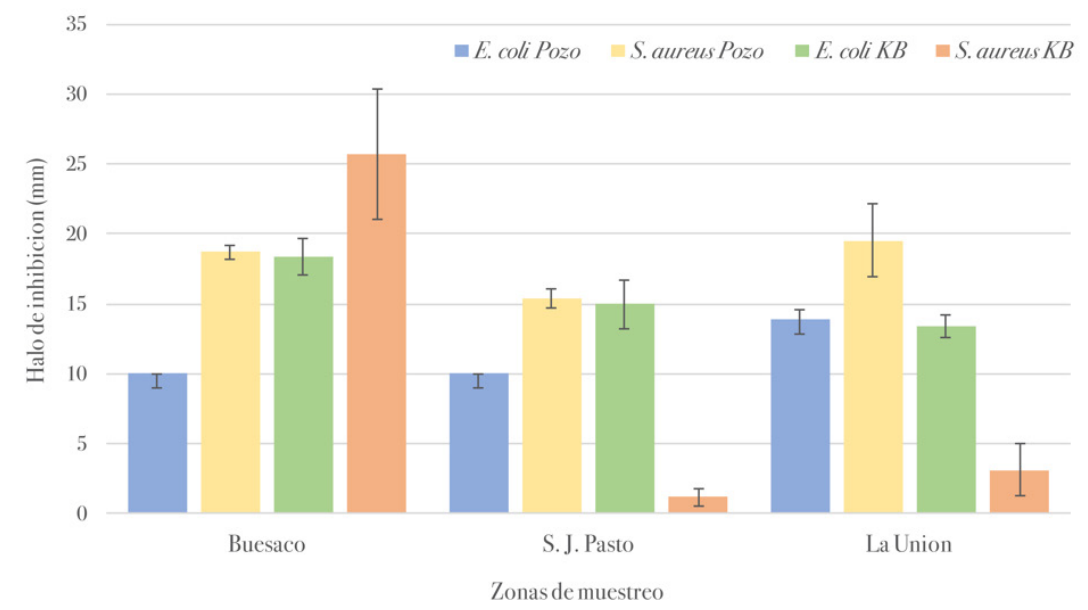

Figura 5. Actividad antibacteriana de extractos alcohólicos de propóleos de Nariño frente a E. coli (ATCC 9.341) y S. aureus (ATCC 25.923) métodos de Kirby-Bauer y difusión en pozos

El contenido de resinas observado en las muestras de Nariño fue superior a los valores encontrados en las de Atlántico (Rodríguez et al., 2012), Bajo Cauca (Viloria et al., 2012) y La Unión, Antioquia (Palomino et al., 2010). En cuanto a los minerales, los valores observados fueron menores respecto a otros propóleos reportados en la literatura (Salamanca et al., 2004). Los rendimientos de resinas del estudio, son concomitantes con los perfil de fenoles y flavonoides totales (mg/g), y con los reportados por otros investigadores (Delgado et al., 2015; Oliveira et al., 2016).

\section{CONCLUSIONES}

El trabajo permitió identificar y caracterizar tipos de propóleos de tres zonas biogeográficas del departamento de Nariño sobre atributos fisicoquímicos, sensoriales y actividad biológica. La huella digital de los espectros de infrarrojo y los cromatogramas HPTLC y ESI(-)-MS/MS), permitieron clasificar las muestras como tipo-O y tipo-B y categorías III a $\mathrm{V}$, como propios de zonas altoandinas propias de región cafetera. El uso de técnicas espectrofotométricas complementarias y de cromatografía revelaron la presencia de metabolitos secundarios con potencial actividad biológica. El trabajo ha hecho posible demostrar la sensibilidad de los propóleos de Buesano, La Unión y S.J. Pasto, frente a S. aureus y E. coli, en la técnica de difusión en pozo y uso de sensidiscos. Los hallazgos contribuyen al estudio de matrices complejas asociadas al sistema apícola productivo desde matrices de propóleos colombianos. 


\section{AGRADECIMIENTOS}

Los autores expresan gratitud Instituto de Biología de la Universidad de Campinas (Brasil), por el acompañamiento y disposición para la evaluación cromatográfica (HPLC-ESI-MS). A la Facultad Ingenieria y Zootecnia de la Universidad de São Paulo (Pirassununga-Brasil), a los Departamentos de Química de las Universidades de Nariño y Tolima. A los evaluadores y colaboradores permanentes de la Revista Biotecnología en el sector Agropecuario y Agroindustrial por las observaciones y recomendaciones durante el proceso de arbitraje.

\section{REFERENCIAS}

AHMED, R.; TANVIR, E.M.; HOSSEN, M.; AFROZ, R.; AHMMED, I.; RUMPA, N.E.; PAUL, S.; HUA-GAN, S.; SULAIMAN, S.; KHALIL, M. Antioxidant properties and cardioprotective mechanism of Malaysian propolis in rats. Evidence-Based Complementary and Alternative Medicine, n. 5370545, 2017, p. 1-11. https://doi:10.1155/2017/5370545

AL-ANI, I.; ZIMMERMANN, S.; REICHLING, J.; WINK, M. Antimicrobial Activities of European Propolis Collected from Various Geographic Origins Alone and in Combination with Antibiotics. Medicines, v. 5, n. 1, 2018, p. 2-17. https://doi:10.3390/medicines5010002

ANJUM, SI.; ULLAH, A.; KHAN, KA.; ATTAULLAH, M.; KHAN, H.; ALI, H.; BASHIR, M.; TAHIR, M.; ANSARI, M.; GHRAMH, H.; ADGABA, N.; DASH, C. Composition and functional properties of propolis (bee glue): A review. Saudi Journal of Biological Science, v. 26, n. 7, 2019, p. 1695-03. https://doi:10.1016/j.sjbs.2018.08.013

BABA, S.A.; MALIK, S.A. Determination of total phenolic and flavonoid content, antimicrobial and antioxidant activity of a root extract of Arisaema jacquemontii Blume. Journal of Taibah University for Science, v. 9, n. 4, 2015, p. 449-54. https://doi:10.1016/j.jtusci.2014.11.001

BARRERA, E.; GIL, J.; RESTREPO, A.; MOSQUERA, K.; DURANGO, D. A coating of chitosan and propolis extract for the postharvest treatment of papaya (Carica papaya L. cv. Hawaiiana). Revista Facultad Nacional de Agronomia Medellin, v. 68, n. 2, 2015, p. 7667-78. https://doi:10.15446/rfnam.v68n2.50982

BASTOS, E.M.; GUZMÁN, D.; FIGUEROA, J.; TELLO, J.; SCOARIS, D.O. Caracterización antimicrobiana y fisicoquímica de propóleos de Apis mellifera L. (Hymenoptera: Apidae) de la región andina colombiana. Acta Biológica Colombiana, v. 16, n. 1, 2011, p. 175-184. http://www.scielo.org.co/scielo.php?script=sci_ arttext\&pid=S0120-548X2011000100013

BETANCES-SALCEDO, E.; REVILLA, I.; VIVAR-QUINTANA, A.; GONZÁLEZ-MARTÍN, M. Flavonoid and Antioxidant Capacity of Propolis Prediction Using Near Infrared Spectroscopy. Sensors, v. 17, n. 7, 2017, p. 1647. https://doi:10.3390/s17071647

CIFTCI-YILMAZ, S.; AZMAN, Z.N.; KOSEM, K.; GUNDUZ, E.; GRENMAN, RG. Evaluating Antioxidant Capacity of Different Propolis Samples from Konya, Turkey and Their Inhibitory Effect on Head and Neck Cancer Cells. BioRxiv. n., 2017, 183913.

https://doi:10.1101/183913

CUESTA-RUBIO, O.; PICCINELLI, A.L.; CAMPO-FERNÁNDEZ, M.; MÁRQUEZ-HERNÁNDEZ, I.; ROSADO, A., RASTRELLI, L. Chemical characterization of Cuban propolis by HPLC-PDA, HPLC-MS, and NMR: the brown, red, and yellow Cuban varieties of propolis. Journal Agricultural and Food Chemical, v. 55, 2007, p. 7502-09. https://doi:10.1021/jf071296w

CUPULL-SANTANA, R.D.; CORTÉS-RODRÍGUEZ, R.; OLAZÁBAL-MANSO, E.E.; HERNÁNDEZ-MEDINA, C.A. Actividad antifúngica de propóleos obtenidos en tres provincias de Cuba sobre hongos contaminantes en cultivos de tejidos vegetales. Acta Universitaria, v. 23, n. 6, 2013, p. 3-9.

https://www.redalyc.org/articulo.oa?id=41629561001 
DELGADO, A.M.L.; ANDRADE, O.J.; RAMÍREZ, B.C.A. Caracterización fisicoquímica de propóleos colectados en el Bosque La Primavera Zapopan, Jalisco. Revista Mexicana de Ciencias Forestales, v. 6, n. 28, 2015, p. 74-87. http://www.scielo.org.mx/scielo.php?script=sci_arttext\&pid=S2007-11322015000200006

DRESCHER, N.; KLEIN, A.M.; NEUMANN, P.; YAÑEZ, O.; LEONHARDT, S. Inside honeybee hives: Impact of natural propolis on the ectoparasitic mite Varroa destructor and viruses. Insects, v. 8, n. 15, 2017, p. 1-18. https://doi:10.3390/insects8010015

EL-GUENDOUZ, S.; LYOUSSI, B.; MIGUEL, M. Insight on Propolis from Mediterranean Countries: Chemical Composition, Biological Activities and Application Fields. Chemistry \& Biodiversity, v. 16, 2019, p. 1-35. https://doi:10.1002/cbdv.201900094

GUZELMERIC, E.; RISTIVOJEVIĆ, P.; TRIFKOVIĆ, J.; DASTAN, T.; YILMAZ, O.; CENGIZ, O.; YESILADA, E. Authentication of Turkish propolis through HPTLC fingerprints combined with multivariate analysis and palynological data and their comparative antioxidant activity. LWT-Food Science and Technology, v. 87, 2018, p. 23-32. https://doi:10.1016/j.lwt.2017.08.060

HERRERA, L.V.; PISCIOTTI, O.M.; RAMOS, M.O.; NEIRA, F.L.; SOTO, S.J. Actividad antimicrobiana in vitro de un propóleo de Santander sobre Enterococcus faecalis. Revista Ustasalud, v. 11, 2012, p. 73-78. https://doi:10.15332/us.v11i2.1119

HOCHHEIM, S.; GUEDES, A.; FACCIN-GALHARDI, L.; RECHENCHOSKI, D.Z.; NOZAWA, C.; LINHARES, R.E.; DA SILVA FILHO, H.; RAU, M.; SIEBERT, D.; MICKE, G.; MENDES DE CORDOVA, C. Determination of phenolic profile by HPLC-ESI-MS/MS, antioxidant activity, in vitro cytotoxicity and anti-herpetic activity of propolis from the Brazilian native bee Melipona quadrifasciata. Brazilian Journal of Pharmacognosy, $v$. 29, n. 3, 2019, p. 339-50. https://doi:10.1016/j.bjp.2018.12.010

KASIOTIS, K.M.; ANASTASIADOU, P.; PAPADOPOULOS, A.; MACHERA, K. Revisiting Greek propolis: chromatographic analysis and antioxidant activity study. PloS One, v. 12, n. 1, 2017, p. 1-27. https://doi:10.1371/journal.pone.0170077

LÓPEZ, B.G.C.; SCHMIDT, E.M.; EBERLIN, M.N.; SAWAYA, A.C.H.F. Phytochemical markers of different types of red propolis. Food Chemistry, v. 146, 2014, p. 174-180. https://doi:10.1016/j.foodchem.2013.09.063

MARTÍNEZ, J.; GARCÍA, C.; DURANGO, D.; GIL, J. Caracterización de propóleos provenientes del municipio de Caldas obtenido por dos métodos de recolección. Revista MVZ Córdoba, v. 17, n. 1, 2012, p. 2861-69. https://doi:10.21897/rmvz.254

MEILGAARD, M.; CIVILLE, G.V.; CARR, B.T. Sensory Evaluation Techniques. 4th ed. Boca Raton (USA): CRC Press; 2015.

https://books.google.com.co/books?hl=es\&lr=\&id=IGjdCgAAQBAJ\&oi=fnd\&pg=PP1\&dq=Sensory+Evaluation+Techniques.+4th+ed.\&ots=FSCgXBCDU9\&sig=XVIpG4_vVbGOcOma8MAOeZ9QVeY\#v=0nepage\&q=Sensory\%20Evaluation\%20Techniques.\%204th\%20ed.\&f=false

MIYATAKA, H.; NISHIKI, M.; MATSUMOTO, H.; FUJIMOTO, T.; MATSUKA, M.; SATOH, T. Evaluation of Propolis. I. Evaluation of Brazilian and Chinese Propolis by Enzymatic and Physico-Chemical Methods. Biological and Pharmaceutical Bulletin, v. 20, n. 5, 1997, p. 496-501. https://doi:10.1248/bpb.20.496

OLIVEIRA, R.N.; MANCINI, M.C.; DE OLIVEIRA, F.C.S.; PASSOS, T.M.; QUILTY, B.; DA THIRÉ, R.M.; Mc GUINNESS. FTIR analysis and quantification of phenols and flavonoids of five commercially available plants extracts used in wound healing. Matéria (Rio J), v. 21, n. 3, 2016, p. 767-79. https://doi:10.1590/S1517-707620160003.0072

OMAR, R.; IGOLI, J.O.; ZHANG, T.; GRAY, A.I.; EBILOMA, G.U.; CLEMENTS, C.J.; FEARNLEY, J.; EBEL, R.; PAGET, T.; DE KONING, H.; WATSON, D. The chemical characterization of Nigerian propolis samples and their activity against Trypanosoma brucei. Scientific Report, v. 7, n. 923, 2017, p. 1-10. https://doi:10.1038/s41598-017-01038-2 
OSORIO, T.M.; SALAMANCA, G.G. Actividad biológica del propóleo. En Origen, naturaleza, propiedades fisicoquímicas y valor terapéutico del propóleo. 1 ed. Ibagué (Colombia): Sello editorial de la Universidad del Tolima, 2017. p. 287-323.

https://www.researchgate.net/publication/328346989_Origen_naturaleza_propiedades_ fisicoquimicas_y_valor_terapeutico_del_propoleo

PALOMINO, L.; MARTÍNEZ, J.; GARCÍA, C.; GIL, J.; DURANGO, D. Caracterización Fisicoquímica y Actividad Antimicrobiana del Propóleos en el Municipio de La Unión (Antioquia, Colombia). Revista Facultad Nacional de Agronomía Medellín, v. 63, 2010, p. 5373-83.

http://www.scielo.org.co/pdf/rfnam/v63n1/a13v63n01.pdf

PRZYBYŁEK, I.; KARPIŃSKI, T.M. Antibacterial Properties of Propolis. Molecules, v. 24, n. 2047, 2019, p. 1-17. https://doi:10.3390/molecules24112047

RODRÍGUEZ, Y.; SÁNCHEZ-CATALÁN, F.; ROJANO, B.; DURANGO, D.; GIL, J.; MARÍN-LOAIZA, J. Caracterización fisicoquímica y evaluación de la actividad antioxidante de propóleos recolectados en el departamento del Atlántico, Colombia. Revista UDCA Actualidad \& Divulgación Científica, v. 15, n. 2, 2012, p. 303-311. http://www.scielo.org.co/scielo.php?pid=S0123-42262012000200007\&script=sci_abstract\&tlng=es

SALAMANCA, G.G.; OSORIO, T.M. New insular red propolis from Colombia: botanical origin, biological and chemical markers. En: 30th International Symposium on the Chemistry of Natural Products and the $10^{\text {th }}$ International Congress On Biodiversity. Atenas (Grecia): 2018.

https://cdn.technologynetworks.com/ep/pdfs/new-insular-red-propolis-from-colombia-botanical-originbiological-and-chemical-markers.pdf

SALAMANCA, G.G.; RAMÍREZ, C.; RUBIANO, L. Contenido mineral de los propóleos colectados en algunas zonas biogeográficas colombianas. Revista Institucional Universidad Tecnológica del Chocó, v. 20, n. 1, 2004, p. 79-85.

SALAMANCA, G.G. Origen, naturaleza, propiedades fisicoquímicas y valor terapéutico del propóleo. 1 ed. Ibagué (Colombia): Sello editorial Universidad del Tolima, 2017.

SAWAYA, A.C.H.F.; BARBOSA-DA SILVA-CUNHA, I.; MARCUCCI, M.C. Analytical methods applied to diverse types of Brazilian propolis. Chemistry Central Journal, v. 5, n. 27, 2011, p. 1-10. https://www.ncbi.nlm.nih.gov/pmc/articles/PMC3123264/pdf/1752-153X-5-27.pdf

SEIBERT, J.B.; BAUTISTA-SILVA, J.P.; AMPARO, T.R.; PETIT, A.; PERVIER, P.; DOS SANTOS ALMEIDA, J.C.; AZEVEDO, M.; SILVEIRA, B.; BRANDÃO, G.; DE SOUZA, G.; TEIXEIRA, L.; DOS SANTOS, O. Development of propolis nanoemulsion with antioxidant and antimicrobial activity for use as a potential natural preservative. Food Chemistry, v. 287, 2019, p. 61-67. https://doi:10.1016/j.foodchem.2019.02.078

SELVARAJ, R.; SIVAKUMARI, K.; FLORA-PRIYADARSHINI, J.; ASHOK, K.; JAYAPRAKASH, P.; RAJESH, S. Phytochemical profiling and antibacterial activity of propolis. International Journal of Scientific Research, v. 7, n. 6, 2018, p. 373-376. https://www.researchgate.net/publication/330170066_PHYTOCHEMICAL_PROFILING_AND_ ANTIBACTERIAL_ACTIVITY_OF_PROPOLIS

SFORCIN, J.M. Biological properties and therapeutic applications of propolis. Phytotheraphy Research, v. 30, 2016, p. 894-905. https://doi:10.1002/ptr.5605

SHAWKY, E.; IBRAHIM, R.S. Bioprofiling for the quality control of Egyptian propolis using an integrated NIR-HPTLC-image analysis strategy. Journal of Chromatography B: Biomedical and Applications, v. 1095, 2018, p. 75-86. https://doi:10.1016/j.jchromb.2018.07.029

TALERO, C.; HERNÁNDEZ, D.; FIGUEROA, J. Calidad microbiológica de propóleo crudo y sólidos solubles de extractos de propóleo de Apis mellifera en Colombia. Revista Medicina Veterinaria y Zootecnia, v. 59, n. 2, 2012, p. 109-118.

http://www.scielo.org.co/scielo.php?pid=S0120-29522012000200005\&script=sci_abstract\&tlng=es 
TOUZANI, S.; EMBASLAT, W.; IMTARA, H.; KMAIL, A.; KADAN, S.; ZAID, H.; ELARABI, I.; BADIAA, L.; SAAD, B. In vitro evaluation of the potential use of propolis as a multitarget therapeutic product: Physicochemical properties, chemical composition, and inmmunomodulatory, antibacterial, and anticancer properties. BioMed Research International, v. 2019, 2019, p. 1-11. https://doi.org/10.1155/2019/4836378

VEIGA, R.S.; DE MENDONÇA, S.; MENDES, P.B.; PAULINO, N.; MIMICA, M.J.; LAGAREIRO NETTO, A.A.; LIRA, I.S.; LOPEZ, B.G.; NEGRÃO, V.; MARCUCCI, M.C. Artepillin C and phenolic compounds responsible for antimicrobial and antioxidant activity of green propolis and Baccharis dracunculifolia DC. Journal of Applied Microbiology, v. 122, n. 4, 2017, p. 911-20. https://doi:10.1111/jam.13400

VELASQUEZ, B.D.; MONTENEGRO, S.P. Actividad antimicrobiana de extractos etanólicos de propóleos obtenidos de Apis mellifera. Revista de Investigación Agraria y Ambiental, v. 8, n. 1, 2017, p. 185-93. https://doi:10.22490/21456453.1848

VELOZ, J.J.; ALVEAR, M.; SALAZAR, L.A. Antimicrobial and antibiofilm activity against Streptococcus mutans of individual and mixtures of the main polyphenolic compounds found in Chilean propolis. BioMed Research International, v. 7602343, 2019, p. 1-7. https://doi:10.1155/2019/7602343

VILORIA, J.; GIL, J.; DURANGO, D.; GARCÍA, C. Caracterización fisicoquímica del propóleo de la Región del Bajo Cauca Antioqueño (Antioquia, Colombia). Biotecnología en el Sector Agropecuario y Agroindustrial, v. 10, 2012, p. 76-85. http://www.scielo.org.co/scielo.php?pid=S1692-35612012000100010\&script=sci_abstract\&tlng=es

ZAINULLIN, R.A.; KUNAKOVA, R.V.; GAREEV, V.F.; GALYAUTDINOV, I.V.; SADRETDINOVA, Z.R.; MUSLIMOV, Z.S.; ODINOKIV, N. Flavanones and flavones from ashkir propolis. Chemistry of Natural Compounds, v. 54 , n. 5, 2018, p. 975-77. https://doi:10.1007/s10600-018-2526-5 